\title{
Clinical features and outcomes of patients with drug-induced autoimmune hepatitis: A retrospective cohort study
}

\author{
Anna Licata ${ }^{a}, *$ Marcello Maida $^{a}$, Daniela Cabibi ${ }^{b}$, Giuseppe Butera ${ }^{a}$, Fabio S. Macaluso ${ }^{a}$, \\ Nicola Alessi ${ }^{a}$, Calogero Caruso ${ }^{c}$, Antonio Craxì ${ }^{a}$, Piero L. Almasio ${ }^{a}$ \\ a Section of Gastroenterology, Di.Bi.M.I.S., University of Palermo, Italy \\ ${ }^{b}$ Section of Anatomical Pathology, University of Palermo, Italy \\ c Section of Clinical Pathology, University of Palermo, Italy
}

\section{A R T I C L E I N F O}

\section{Article history:}

Received 4 March 2014

Accepted 19 August 2014

Available online $\mathrm{xxx}$

\section{Keywords:}

Autoimmunity

Drug-induced liver injury

Human leucocyte antigens

Liver biopsy

\begin{abstract}
A B S T R A C T
Background: Drugs and herbal products can induce autoimmune hepatitis. We assessed frequency and clinical outcomes of patients suffering from drug-induced autoimmune hepatitis.

Methods: All patients with drug-induced liver injury admitted between 2000 and 2011 were retrospectively studied. Diagnoses of drug-induced autoimmune hepatitis and idiopathic autoimmune hepatitis were made according to simplified criteria. After discharge, all patients had regular follow-up and were contacted to update outcomes.

Results: Among 10,270 in-hospital patients, 136 (1.3\%) were diagnosed with drug-induced liver injury. Among them, $12(8.8 \%)$ were diagnosed as drug-induced autoimmune hepatitis $(41.7 \%$ males, age range 17-73); 8 (66.7\%) were with jaundice at admission. Liver biopsies showed a pattern compatible with drug-induced autoimmune hepatitis, featured by severe portal inflammation and lymphoplasmacytic infiltrate. Drug-induced autoimmune hepatitis group had a shorter duration of drug intake, and higher values of transaminases and gamma globulins. All patients received immunosuppressive therapy with subsequent clinical remission, and five achieved a steroid-free long-term remission.

Conclusions: A diagnosis of drug-induced autoimmune hepatitis was quite rare in our cohort, and clinical pattern was similar to idiopathic autoimmune hepatitis. Severe portal inflammation, prominent portalplasma cells, rosette formation and severe focal necrosis were significantly more frequent in drug-induced autoimmune hepatitis as compared to drug-induced liver injury.
\end{abstract}

(c) 2014 Published by Elsevier Ltd on behalf of Editrice Gastroenterologica Italiana S.r.l.

\section{Introduction}

Autoimmune hepatitis (AIH) and drug-induced liver injury (DILI) are two different nosological entities, apparently similar in clinical presentation and immunological pictures, characterized by acute onset at presentation, highly altered liver function tests, and autoantibody positivity. However, autoantibodies in DILI are frequently negative. Diagnosis can be challenging, especially from the histologic point of view, given the crucial role played by the immunologic reaction, featured by interface hepatitis, lymphoplasmacytic infiltrate, rosette formation and cholestasis, thus resembling idiopathic autoimmune hepatitis [1].

\footnotetext{
* Corresponding author at: Section of Gastroenterology, Di.Bi.M.I.S., University of Palermo, Piazza delle Cliniche 2, 90127 Palermo, Italy. Tel.: +39 0916552280; fax: +390916552156

E-mail address: anna.licata@unipa.it (A. Licata).
}

In particular, DILI is a potential complication that can occur with the intake of many medications, herbs and/or dietary supplements. It is also the principal reason of medications withdrawal from the market [2], and the most common cause of fulminant hepatic failure in both the United States and Europe [3,4]. More than 1000 different drugs and herbal products have been described as cause of DILI, with a dose-dependent, or more frequently, an idiosyncratic toxicity. Among them antimicrobials, interferon, infliximab and statins can induce hepatocellular damage that mimics an autoimmune liver injury [5-7], and the same possibility has also been suggested for herbal products, such as Dai-saiko-to and black cohosh $[8,9]$. Recently Björnsson et al. [5] described a well characterized cohort of patients with drug-induced autoimmune hepatitis (DI-AIH) from minocycline or nitrofurantoin. Overall, the histological and serological features of these patients were indistinguishable from those with idiopathic $\mathrm{AIH}$, with the only difference being the favourable clinical outcome for the DI-AIH patients. Because DI-AIH is prevalent in women, as well as shows an acute onset, and no signs of 
chronic liver damage at presentation (unlike idiopathic $\mathrm{AIH}$ ), clinical evaluation is the best option for diagnosing this condition [10].

However, because of the lack of pathognomonic features and the resulting absence of specific diagnostic criteria, there is no consensus on the adequate terminology for defining the varying clinical patterns of DILI $[11,12]$. Recently, Weiler-Normann and Schramm [13], attempting to define the relationship between DILI and autoimmune hepatitis, identified three different clinical entities, named AIH with DILI, drug-induced AIH and immunemediated DILI.

In this study, we aimed to assess the frequency, clinical course, histological features and outcomes of a cohort of patients suffering from DI-AIH by drugs or herbal products.

\section{Patients and methods}

This study was carried out analysing all cases of DILI systematically collected at our Liver Unit, a tertiary referral centre for chronic liver diseases. We retrospectively reviewed all clinical records of patients admitted to our Unit between January 2000 and December 2011. All patients with a diagnosis of DILI were assessed with clinical, laboratory and imaging tests, together with liver histology as it related to their clinical outcome. A hepatocellular pattern of damage was defined as chronic if liver tests showed a persistent abnormality more than 3 months after stopping drug therapy. In the case of cholestatic/mixed type of liver injury, the abnormality needed to be persistent for more than 6 months following drug withdrawal. Cases were defined as resolved when liver function tests (LFTs) returned within laboratory reference ranges within this period of time [12]. For all patients, a detailed history was obtained concerning antecedents of liver or biliary disease, drug addiction and/or alcohol abuse, transfusion of blood products, or surgery within 6 months preceding the onset of liver disease. All patients underwent radiographic tests to rule out obstructive aetiologies of liver injury.

A computerized database was created, and reported age, gender, clinical features at onset, laboratory results, other concomitant disease, suspected drugs and follow-up. All patients were tested for hepatitis A, B, C viruses, Epstein-Barr virus and cytomegalovirus serology. In addition, a non-organ-specific autoantibody (NOSA) profile was created, and included anti-mitochondrial antibody (AMA), anti-smooth muscle antibody (SMA), anti-liver-kidney microsomal antibody (LKM).

Only cases considered drug-related according to the clinical judgement of experts were assessed by CIOMS/RUCAM (Council for International Organizations of Medical Sciences/Roussel Uclaf Causality Assessment Method scale) [11]. Cases classified as definite, highly probable, probable, or possible, were entered into the database.

Diagnosis of DILI was performed according to Consensus criteria demonstrating the chronological relationship between drug intake and onset of hepatitis and the presence of any of the following: (i) $\geq 5 \times$ the upper limit of normal (ULN) for alanine aminotransferase (ALT); (ii) $\geq 2 \times$ ULN for alkaline phosphatase (ALP); (iii) $\geq 3 \times$ ULN for ALT concentration and simultaneous elevation of bilirubin concentration $\geq 2 \times$ ULN [12]. Pattern of liver injury was assessed using $R$ value where $R=($ ALT/ULN)/(ALP/ULN). Hepatocellular pattern was defined for $R$ value $\geq 5$, mixed pattern for $R$ value $>2$ and $<5$, cholestatic pattern for $R$ value $\leq 2$. All liver tests used for the classification of liver damage were the first blood test available at the time liver injury was suspected.

The diagnosis of DI-AIH was performed according to Consensus criteria demonstrating the presence of the following: (i) a score $\geq 6$ points on simplified diagnostic criteria for AIH [14] (using a value $>6$ as cut-off for definition of probable $\mathrm{AIH}$ ); (ii) injury resolution on withdrawal of medication that triggered the $\mathrm{AIH}$, with or without immunosuppressive therapy to induce remission; (iii) no relapse within a period of 1 year after withdrawal of all immunosuppressants [12]. A liver biopsy with a detailed histological evaluation was performed in 44/136 patients, including, among all, the suspected cases of $\mathrm{AIH}$, and all slides were evaluated by an expert pathologist (DC).

In 5 out of 12 patients with diagnosis of DI-AIH who agreed to, low resolution HLA genotyping was finally performed using standard procedures with the One Lambda kit (Lagitre, Milan, Italy). All patients had regular follow-up visits every 3 months after discharge, and were re-contacted by phone to update their clinical outcomes.

\subsection{Statistical analysis}

Data are reported as medians with interquartile ranges or frequencies. Median values of the variables were compared among different groups with the Kruskall-Wallis test. A chi-square test was used to compare categorical variables. A $p$ value of less than 0.05 was considered statistically significant. All $p$ values were twotailed. All analyses were performed using SPSS v. 20.0 statistical package for MacIntosh (SPSS Inc., Chicago, USA).

\section{Results}

Of the 10,270 patients admitted to our Unit between January 2000 and December 2011, only $136(1.3 \%)$ were discharged with a diagnosis of DILI (Fig. 1). Among them, 52/136 (38.2\%) were positive for NOSAs, while 12/136 (8.8\%) were diagnosed as having a DI-AIH based on clinical history, autoantibody pattern and histological examination. Demographic, clinical, laboratory, histological features and outcomes of each patient are shown in Supplementary Table S1. None of the patients showed evidence of autoimmune diseases, or developed manifestations of extra-hepatic autoimmunity.

Of the patients with a diagnosis of DI-AIH, 5/12 (41.7\%) were male and $7 / 12$ (58.3\%) female, with a mean age of 56.5 years (range 18 to 73). In each case, liver damage developed in temporal association with the intake of pharmaceuticals at recommended doses. Nine of the 12 (75\%) presented with acute liver injury, and all were with jaundice. Time to onset of liver injury and duration of therapy ranged from 3 days to 8 weeks. Only 3 patients did not have an $\mathrm{AIH}$ score $\geq 6$ points. For these, the diagnosis of DI-AIH was based on the clinical judgement of the treating physician.

Ten of the 12 patients ( $83.3 \%$ ) showed a pattern of liver damage of hepatocellular type, one was cholestatic, and the other mixed. The hepatocellular liver injury was caused by non-steroidal antiinflammatory drugs (NSAIDs) (nimesulide/ketoprofen) in 5 cases, and by antimicrobial drugs (amoxicillin-clavulanate/ceftriaxone) in 3. In one case, the etiologic agent was green tea, and in one was dimethoate, an organophosphate insecticide. Cholestatic liver injury was detected in one patient, who was exposed to nimesulide and ketoprofen; a mixed liver damage observed following the intake of Hypericum perforatum.

\subsection{Clinical course of patients with DI-AIH}

The mean time of follow-up was 26 months (range 12-84). All patients started a corticosteroid therapy with tapering doses, and in 7 of them azathioprine was also added as a corticosteroid-sparing agent. After 15 months, all patients were in remission, with normal ALT (Fig. 2). Immunosuppressive treatment was stopped in 5 patients after two years, and none of them showed a relapse after steroid withdrawal. There was no liver-related death, and none developed cirrhosis or required liver transplantation. All 


\section{0,270 patients admitted to Gastroenterology and Hepatology Unit}

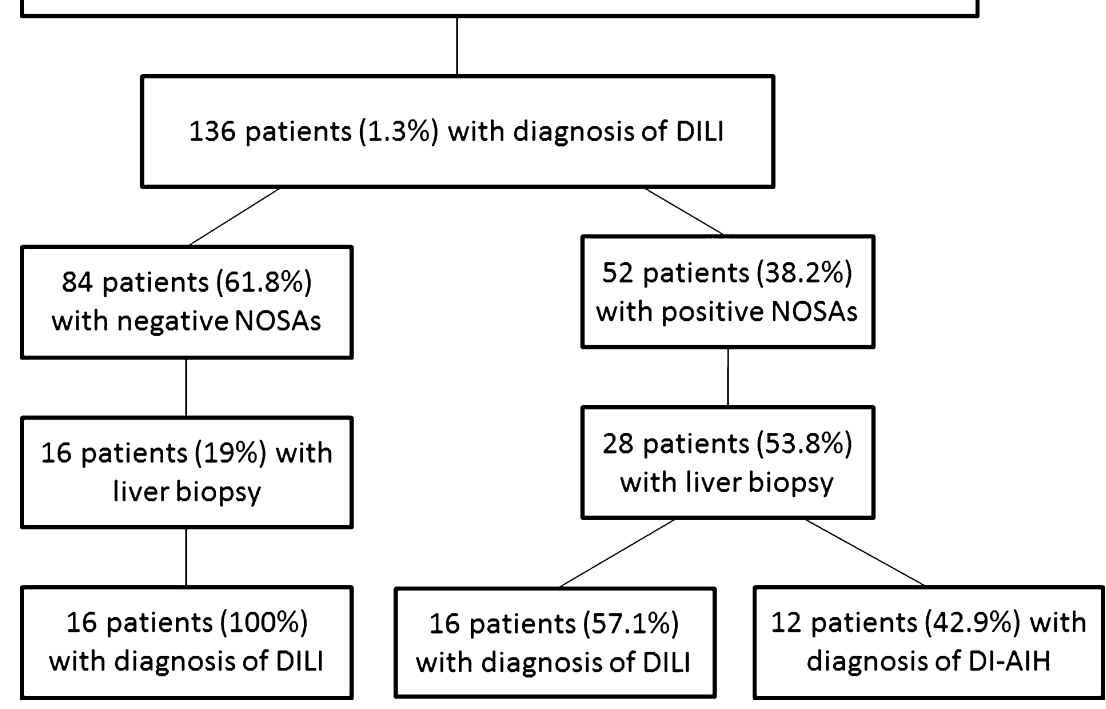

Fig. 1. Flow chart of the study. DI-AIH, drug-induced autoimmune hepatitis; DILI, drug-induced liver injury; NOSA, non-organ-specific autoantibody.

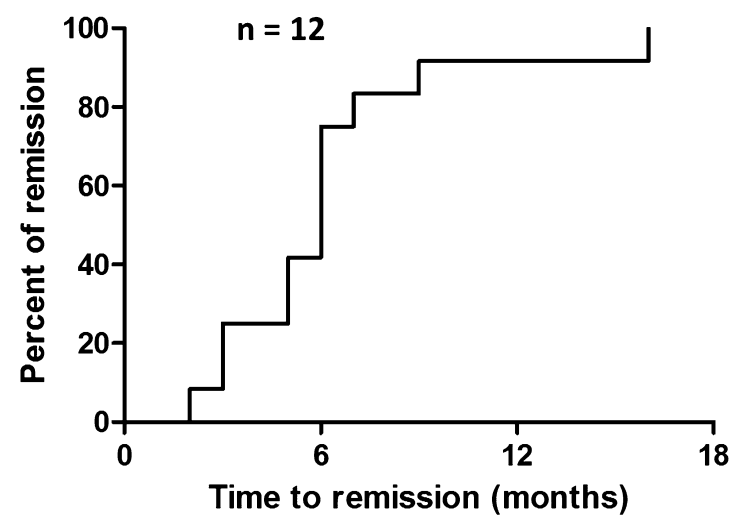

Fig. 2. Time to ALT normalization after starting immunosuppressive therapy in patients with drug-induced autoimmune hepatitis (number of patients $=12$ ).

patients with DI-AIH were still alive at the last follow-up visit, and none of them developed extra-hepatic manifestations as a marker of autoimmune disease. In patient 1 , a new hepatitis flare was observed on maintenance of low-dose prednisone therapy, and in patient 10 , interruption of treatment led to a new rise in cytolytic activity, indicating that the need of long term immunosuppressive therapy (Supplementary Table S1).

\subsection{Comparison of DI-AIH with DILI}

Table 1 shows clinical and laboratory characteristics of patients with DI-AIH compared with those patients with NOSA-positive DILI and without features of autoimmune disease, and NOSAnegative DILI patients. DI-AIH patients showed a significantly shorter duration of drug intake and higher values of transaminases and gamma-globulins.

DI-AIH liver biopsies were separately compared with 16 liver specimens of NOSA-positive and with 16 liver specimens of NOSA-negative DILI patients considering all the histological features. Comparing DI-AIH and DILI/NOSAs negative specimens by univariate analysis, severe portal inflammation $\geq$ grade 2 $(p=0.01)$, prominent portal-plasma cells $(p=0.01)$, rosette formation $(p=0.003)$, any level of fibrosis $\geq$ grade $1(p=0.009)$ and severe focal necrosis $\geq$ grade $4(p=0.02)$ were more prevalent in DI-AIH group (Table 2).

Table 1

Demographic, clinical and laboratory findings of patients who developed drug-induced liver injury according to different autoimmune pattern.

\begin{tabular}{|c|c|c|c|c|}
\hline Variables & $\begin{array}{l}\text { DI-AIH } \\
(n=12)\end{array}$ & $\begin{array}{l}\text { DILI/NOSAs negative } \\
(n=84)\end{array}$ & $\begin{array}{l}\text { DILI/NOSAs positive } \\
(n=40)\end{array}$ & $p$ \\
\hline Age, years (median, IQR) & $56.5(42.7-64.7)$ & $54.0(35.0-68.0)$ & $56.0(46.0-66.5)$ & 0.8 \\
\hline Male gender & $5 / 12(41.7 \%)$ & $44 / 84(52.4 \%)$ & $17 / 40(42.5 \%)$ & 0.5 \\
\hline Duration of drug intake, days (median, IQR) & $4(1.0-9.2)$ & $10(5.0-30.0)$ & $7(6.0-50.0)$ & 0.02 \\
\hline Latency, weeks (median, IQR) & $4(1.0-9.2)$ & $15(10.0-60.0)$ & $15(10.5-37.5)$ & 0.7 \\
\hline Jaundice at onset & $8 / 12(66.6 \%)$ & $40 / 84(47.6 \%)$ & $16 / 40(40.0 \%)$ & 0.2 \\
\hline Hepatic encephalopathy at diagnosis & $0 / 12(0 \%)$ & $3 / 84(3.6 \%)$ & $3 / 40(7.5 \%)$ & 0.4 \\
\hline AST, $\times$ ULN (median, IQR) & $18.0(10.0-50.0)$ & $5.0(2.0-15.0)$ & $6.5(3.0-20.0)$ & 0.04 \\
\hline ALT, $\times$ ULN (median, IQR) & $25.0(11.0-30.0)$ & $7.0(4.0-20.0)$ & $9.5(13.1-24.5)$ & 0.07 \\
\hline GGT, $\times$ ULN (median, IQR) & $3.5(1.2-6.0)$ & $5.0(2.0-12.0)$ & $4.0(2.2-9.7)$ & 0.5 \\
\hline ALP,$\times$ ULN (median, IQR) & $1.5(1.0-2.4)$ & $2.0(1.0-4.0)$ & $1.5(1.0-2.0)$ & 0.1 \\
\hline Total bilirubin, mg/dl (median, IQR) & $5.5(1.0-14.7)$ & $2.0(1.0-8.0)$ & $1.6(0.8-9.3)$ & 0.5 \\
\hline Gamma globulins (g/L) (median, IQR) & $18.0(1.45-22.0)$ & $10.4(8.5-13.7)$ & $13.6(10.9-21.4)$ & 0.001 \\
\hline Platelet count, $\times 10^{3} / \mathrm{mmc}$ (median, IQR) & $202.0(160.0-237.5)$ & $224.0(153.0-305.0)$ & $212.5(157.0-294.0)$ & 0.7 \\
\hline Eosinophil count/mmc, (median, IQR) & $85(22.5-195.0)$ & $141(70.0-240.7)$ & $100(30.0-222.5)$ & 0.4 \\
\hline Liver stiffness, kPa (median, IQR) & $14.5(7.2-23.3)$ & $6.0(5.0-11.2)$ & $8.7(5.4-15.9)$ & 0.1 \\
\hline
\end{tabular}

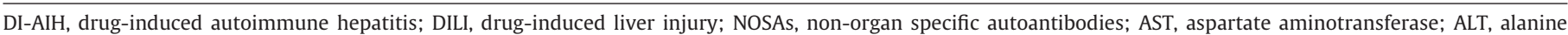
aminotransferase; GGT, gamma-glutamyl transferase; ALP, alkaline phosphatase; IQR, interquartile range; ULN, upper limit of normal; KPa, kiloPascal. 
Table 2

Histological features of liver biopsies in patients with drug-induced autoimmune hepatitis and drug-induced liver injury NOSAs negative.

\begin{tabular}{lccl}
\hline Histological features & DI-AIH & $\begin{array}{l}\text { DILI/NOSAs } \\
\text { negative } \\
(n=16)\end{array}$ & $p$ \\
\hline Severe portal inflammation ( $\geq$ grade 2$)$ & $100 \%$ & $62.5 \%$ & 0.01 \\
Prominent intra-acinar lymphocytes & $91.6 \%$ & $75.0 \%$ & 0.2 \\
Prominent intra-acinar eosinophils & $50.0 \%$ & $31.3 \%$ & 0.3 \\
Cholestasis-canalicular & $66.6 \%$ & $37.5 \%$ & 0.1 \\
Prominent portal-plasma cells & $58.3 \%$ & $12.5 \%$ & 0.01 \\
Rosette formation & $66.7 \%$ & $6.3 \%$ & 0.003 \\
Any level of fibrosis ( $\geq$ grade 1) & $17.0 \%$ & $25.0 \%$ & 0.1 \\
Prominent port-neutrophils & $75.0 \%$ & $62.5 \%$ & 0.4 \\
Hepatocellular cholestasis & $58.3 \%$ & $50.0 \%$ & 0.6 \\
Severe focal necrosis ( $\geq$ grade 4) & $66.6 \%$ & $25.0 \%$ & 0.02 \\
\hline
\end{tabular}

DI-AIH, drug-induced autoimmune hepatitis; DILI, drug-induced liver injury; NOSAs, non-organ specific autoantibodies.

Table 3

Histological features of liver biopsies in patients with drug-induced autoimmune hepatitis and drug-induced liver injury NOSAs positive.

\begin{tabular}{lccl}
\hline Histological features & DI-AIH & $\begin{array}{l}\text { DILI/NOSAs } \\
\text { positive } \\
(n=16)\end{array}$ & $p$ \\
\hline Severe portal inflammation ( $\geq$ grade 2$)$ & $100 \%$ & $56.2 \%$ & 0.008 \\
Prominent intra-acinar lymphocytes & $91.6 \%$ & $56.2 \%$ & 0.04 \\
Prominent intra-acinar eosinophils & $50.0 \%$ & $50.0 \%$ & 0.1 \\
Cholestasis-canalicular & $66.6 \%$ & $34.3 \%$ & 0.06 \\
Prominent portal-plasma cells & $58.3 \%$ & $6.3 \%$ & 0.003 \\
Rosette formation & $66.7 \%$ & $12.5 \%$ & 0.01 \\
Any level of fibrosis ( $\geq$ grade 1) & $17.0 \%$ & $43.7 \%$ & 0.09 \\
Prominent port-neutrophils & $75.0 \%$ & $68.7 \%$ & 0.7 \\
Hepatocellular cholestasis & $58.3 \%$ & $34.3 \%$ & 0.1 \\
Severe focal necrosis ( $\geq$ grade 4) & $66.6 \%$ & $6.3 \%$ & 0.001 \\
\hline
\end{tabular}

DI-AIH, drug-induced autoimmune hepatitis; DILI, drug-induced liver injury; NOSAs, non-organ specific autoantibodies.

The comparison of DI-AIH with DILI/NOSAs positive specimens by univariate analysis showed that severe portal inflammation $\geq$ grade $2(p=0.008)$, prominent intra-acinar lymphocytes $(p=0.04)$, prominent portal-plasma cells $(p=0.003)$, rosette formation $(p=0.01)$ and severe focal necrosis $\geq$ grade $4(p=0.001)$ were more prevalent in DI-AIH group (Table 3 ). In both cases severe portal inflammation, prominent portal-plasma cells, rosette formation and severe focal necrosis were significantly more frequent in DI-AIH group compared to DILI (either positive or negative NOSAs).

\section{Discussion}

Clinical assessment of patients with elevated LFTs after exposure to medication is necessary for a prognosis, because withdrawal of the culprit drug is not always associated with complete recovery. In this setting, assessing the possibility of DI-AIH, a clinical entity in which liver injury is associated with the appearance of autoantibodies and histologic features of both autoimmune disease and DILI, is a reliable option, to be taken into consideration as a diagnostic approach [1].

Attempting to evaluate the frequency of autoimmune disease after DILI, we searched our DILI cohort of 136 patients, consecutively diagnosed and followed at our Liver Unit since January 2000 , for whom demographic data, clinical and biochemical features and outcomes are periodically updated. Twenty-five percent had pre-existing compensated chronic viral hepatitis or cirrhosis. A hepatocellular pattern of DILI was more commonly observed (53.2\%), followed by cholestatic (26.7\%) and mixed pattern $(20.1 \%)$.
About $40 \%$ of our DILI patients were NOSA-positive. Of these patients, only $12(8.8 \%)$ shared clinical and histological stigmata of autoimmune hepatitis, suggesting that DI-AIH represents a quite rare clinical condition in the setting of DILI. Comparing the clinical features of DI-AIH, DILI (both positive and negative NOSAs) only ALT, and gamma globulin values were, as expected, significantly higher in DI-AIH group.

A direct and peculiar characteristic of our cases is the high values of liver stiffness compared with DILI patients. The use of TE to estimate liver fibrosis has been repeatedly validated in different settings even if its performance seems to be affected by several factors, such as alanine aminotransferase flares and severe liver necro-inflammation, hepatic congestion, extrahepatic cholestasis and high body mass index (BMI) [15]. Even if Fibroscan ${ }^{\circledR}$ has not yet been validated for DILI, we are confident enough that high levels of liver stiffness may be the result of an overestimation as a consequence of intense necro-inflammatory activity, which has been described for acute viral hepatitis [16].

However, the key clinical question is the differential diagnosis between DI-AIH and DILI, especially in the presence of NOSAs positivity [17]. Up to now, differential diagnosis is challenging because of the lack of pathognomonic features and the resulting absence of enough specific diagnostic criteria.

In this study we compared the histological features of DI-AIH vs DILI/NOSAs negative and of DI-AIH vs DILI/NOSAs positive, in order to assess the possible presence of significant differences between the 3 groups. Although evaluated in a different setting (distinction of AIH vs. idiopathic DILI), the presence of histological criteria described by Suzuki et al. [1] was also investigated in all the histological specimens, in order to assess their discrimination power also in the differential diagnosis of DI-AIH versus DILI.

In both comparative analyses (Tables 2 and 3) we found that severe portal inflammation, prominent portal-plasma cells, rosette formation and severe focal necrosis were more prevalent in DIAIH group. These same features were found to be more frequent in I-AIH by Suzuki et al. when compared to DILI. Our result seems to confirm that these histological features are also frequent in DIAIH when compared to DILI, showing how these could be useful in distinguishing the histologic picture of $\mathrm{AIH}$ (both idiopathic and drug-induced) from DILI.

One more point that is challenging is to distinguish latent idiopathic AIH unmasked by pharmaceuticals from DI-AIH. This distinction is neither useful nor feasible in the majority of cases always, due to the absence of specific useful features, as pointed out by Björnsson et al. [4], Suzuki et al. [1], and recently stressed by Weiler-Normann and Schramm [13]. In this setting, the criteria that argue for DI-AIH are the absence of fibrosis at histological examination and the absence of recurrence after discontinuation of immunosuppressive therapy. In our cohort, all patients who discontinued therapy (5/12) showed absence of recurrence, and only two patients had mild fibrosis at histological examination. However, as already pointed out, this distinction is not always clinically useful, nor even possible in real practice.

Another challenging point concerns the genetic susceptibility to drug-induced liver damage. Despite the correlation between HLA-DR/DQ status and AIH is well known [18], we have limited knowledge of HLA status and occurrence of DI-AIH. In our series, only 5 of the 12 DI-AIH patients were assessed for HLA. These genotyped patients carried HLA DRB1-03 and/or DRB1-13, known to be associated with autoimmune disease, $[19,20]$. Although the genotype was not performed in all patients, we can speculate that HLA II class type DR/DQ carried by our patients could explain the role of genetic susceptibility to nimesulide, other xenobiotics, as has been recently demonstrated for both HLA I, and II classes to beta-lactams [21-23]. Taken together, these data strengthen a previous hypothesis of autoimmune pathogenesis, in which several environmental 
triggers generate different epitopes, each to be presented by the different HLA-DR molecules [19].

Albeit DI-AIH is a rare condition, distinguishing this clinical entity among the group of DILI is essential for prognosis, and correct choice of treatment options [17]. In absence of consensus agreement on proper treatment, immunosuppression is still recommended for severe disease at onset and subsequent management should be based on individual clinical follow-up. In addition, due to the rarity of this condition, there is no standardized schedule of follow-up. Our experience suggests that close monitoring of LFTs, autoimmune profile and hypergammaglobulinemia may be indicated.

In addition, no clear indications exist in literature regarding discontinuation of treatment in DI-AIH. However it must be noted that, despite the lack of robust data, the suspension of immunosuppressive therapy is suggested by international criteria in order to obtain an additional criterion "a posteriori" for the definition DI$\mathrm{AIH}$, in case of no relapse within a period of 1 year after withdrawal of all immunosuppressants [12]. In our experience, discontinuation of immunosuppressive therapy could be taken into account if the patient obtained a minimum duration of biochemical remission without sign of disease activity after at least 24 months after ALT normalization. In this setting, liver biopsy assessment prior to termination of treatment is the only method by which to ensure full resolution of the disease and therefore it should be always performed [24].

We observed prompt biochemical remission after immunosuppressive therapy in all cases of DI-AIH. In particular, all our patients had a better prognosis and a more complete response to therapy than typical idiopathic AIH patients, in whom, even if there is an initial normalization of LFTs, relapse after withdrawal of immunosuppression is almost the rule [24,25].

All these data support the importance of a correct distinction of DI-AIH in the group of DILI, emphasizing the key role of liver biopsy in the diagnosis, prognosis and follow-up of the disease. In our cohort of patients, we found that severe portal inflammation, prominent intra-acinar lymphocytes, fibrosis and necrosis may be helpful in histological distinction between DIAIH and DILI. This is similar to that described by Suzuki et al. [1] for the distinction between idiopathic AIH and DILI. However, despite some recent evidence, to date no criteria has been successfully validated and the differential diagnosis is still challenging. In this regard, the physician's clinical judgement plays a crucial role in reaching the diagnosis and in establishing a correct management.

\section{Conflict of interest}

None declared.

\section{Appendix A. Supplementary data}

Supplementary data associated with this article can be found, in the online version, at http://dx.doi.org/10.1016/j.dld.2014.08.040.

\section{References}

[1] Suzuki A, Brunt EM, Kleiner DE, et al. The use of liver biopsy evaluation in discrimination of idiopathic autoimmune hepatitis versus drug-induced liver injury. Hepatology 2011;54:931-9.

[2] Navarro VJ, Senior JR. Drug-related hepatotoxicity. New England Journal of Medicine 2006;354:731-9.

[3] Ostapowicz G, Fontana RJ, Schiodt FV, et al., U.S. Acute Liver Failure Study Group. Results of a prospective study of acute liver failure at 17 tertiary care centers in the United States. Annals of Internal Medicine 2002;137:947-54.

[4] Wei G, Bergquist A, Broomé U, et al. Acute liver failure in Sweden: etiology and outcome. Journal of Internal Medicine 2007;262:393-401.

[5] Björnsson E, Talwalkar J, Treeprasertsuk S, et al. Drug-induced autoimmune hepatitis: clinical characteristics and prognosis. Hepatology 2010;51:2040-8.

[6] Germano V, Picchianti Diamanti A, Baccano G, et al. Autoimmune hepatitis associated with infliximab in a patient with psoriatic arthritis. Annals of the Rheumatic Diseases 2005;64:1519-20.

[7] Wolters LM, Van Buuren HR. Rosuvastatin-associated hepatitis with autoimmune features. European Journal of Gastroenterology and Hepatology 2005; 17:589-90.

[8] Kamiyama T, Nouchi T, Kojima S, et al. Autoimmune hepatitis triggered by administration of an herbal medicine. American Journal of Gastroenterology 1997;92:703-4.

[9] Licata A, Macaluso FS, Craxì A. Herbal hepatotoxicity: a hidden epidemic. Internal and Emergency Medicine 2013;8:13-22.

[10] Czaja AJ. Drug-induced autoimmune-like hepatitis. Digestive Diseases and Sciences 2011:56:958-76

[11] Fontana RJ, Seeff LB, Andrade RJ, et al. Standardization of nomenclature and causality assessment in drug-induced liver injury: summary of a clinical research workshop. Hepatology 2010;52:730-42.

[12] Aithal Gp PB, Watkins RJ, Andrade D, et al. Case definition and phenotype standardization in drug-induced liver injury. Clinical Pharmacology and Therapeutics 2011;89:806-15.

[13] Weiler Normann C, Schramm C. Drug-induced liver injury and its relationship between to autoimmune hepatitis. Journal of Hepatology 2011;55:747-9.

[14] Hennes EM, Zeniya M, Czaja AJ, et al. Simplified criteria for the diagnosis of autoimmune hepatitis. Hepatology 2008;48:169-76.

[15] Macaluso FS, Maida M, Cammà C, et al. Steatosis affects the performance of liver stiffness measurement for fibrosis assessment in patients with genotype 1 chronic hepatitis C. Journal of Hepatology 2014, http://dx.doi.org/10.1016/ j.jhep.2014.04.045. pii:S0168-8278(14)00308-0.

[16] Di Marco V, Calvaruso V, Iacò A, et al. Liver stiffness measurement by transient elastography predicts early recovery from acute hepatitis. Gut 2011:60:1023.

[17] Lucena MI, Kaplowitz N, Hallal H, et al. Recurrent drug-induced liver injury (DILI) with different drugs in the Spanish registry: the dilemma of the relationship with autoimmune hepatitis. Journal of Hepatology 2011;55:820-7.

[18] Qiu DK, Ma X. Relationship between human leukocyte antigen-DRB1 and autoimmune hepatitis type I in Chinese patients. Journal of Gastroenterology and Hepatology 2003;18:63-7.

[19] Goldberg AC, Bittencourt PL, Bougin P, et al. Analysis of HLA haplotypes in autoimmune hepatitis type 1: identifying the major susceptibility locus. Human Immunology 2001;62:165-9.

[20] Oliveira LC, Porta G, Marin ML, et al. Autoimmune hepatitis, HLA and extended haplotypes. Autoimmunity Reviews 2011;10:189-93.

[21] Lucena MI, Molokhia M, Shen Y, et al. Susceptibility to amoxicillinclavulanate-induced liver injury is influenced by multiple HLA class I and Il alleles. Gastroenterology 2011;141:338-47.

[22] Donaldson PT, Daly AK, Henderson J, et al. Human leucocyte antigen class II genotype in susceptibility and resistance to co-amoxiclav-induced liver injury. Journal of Hepatology 2010;53:1049-53, http://dx.doi.org/10.1016/ j.jhep.2010.05.03.

[23] Daly AK, Donaldson PT, Bhatnagar P, et al. HLA-B*5701 genotype is a major determinant of drug-induced liver injury due to flucloxacillin. Nature Genetics 2009;41:816-9, http://dx.doi.org/10.1038/ng.379.

[24] Manns MP, Czaja AJ, Gorham JD, et al. Diagnosis and management of autoimmune hepatitis. Hepatology 2010;51:2193-213.

[25] vanGerven NM, Verwer BJ, Witte BI, et al. Relapse is almost universal after withdrawal of immunosuppressive medication in patients with autoimmune hepatitis in remission. Journal of Hepatology 2013;58:141-7. 